\title{
Gear Fault Diagnosis based on Variational Mode Decomposition and Envelope Spectrum
}

\author{
Yingjie Wang ${ }^{1,2, *}$, Wentao $\mathrm{Yu}^{2}$ and Jianyi Qin ${ }^{3}$ \\ ${ }^{1}$ School of Mechanical \& Precision Instrument Engineering, Xi'an University of Technology, Xi'an 710048, China \\ ${ }^{2}$ School of Mechatronics Engineering, Zhongyuan University of Technology, Zhengzhou 450007, China \\ ${ }^{3}$ Electrical Engineering and Information Technology, University of Dortmund, Germany
}

Received 21 July 2018; Accepted 10 October 2018

\begin{abstract}
Multi-component non-stationary vibration signals produced at local gear fault can easily be covered by periodic harmonic signal and strong background noise, thus causing difficulty in selecting gear fault features and diagnosing fault state. To address this issue, a gear fault diagnosis method based on variational mode decomposition (VMD) and envelope spectrum was proposed in this study, which was then employed to select gear-fault features from multi-component vibration signals adaptively, extract fault characteristic frequency, and determine health conditions of the gear. Based on VMD of gear-fault vibration signals in complicated environments, the relationship between center frequency at decomposed intrinsic mode functions (IMFs) and the gear mesh frequency was discussed. The technique used to determine the number of decomposed modes and gear-fault characteristic component was also analyzed. On this basis, the gear fault state was disclosed. The proposed approach was validated by fault simulation and experimental data. Results demonstrate that the number of decomposed modes can influence VMD and the selection of fault characteristic component. The center frequency of IMFs obtained from VMD is compared with the gear mesh frequency. When the difference is lower than a certain threshold, the corresponding IMF is the gear-fault characteristic component, and the number of decomposed modes is simultaneously determined. The fault characteristic frequency is extracted from Hilbert envelope demodulation of characteristic component, which is used to determine the gear fault state. Conclusions obtained in this study can provide important references for the VMD method to facilitate effective selection of fault characteristic components during gear fault diagnosis and offer new insights into this type of diagnosis.
\end{abstract}

Keywords: Variational mode decomposition, Envelope spectrum, Gear, Fault diagnosis

\section{Introduction}

Vehicle gearboxes operate under complicated conditions (e.g., variable loads) for a long time; thus, running fatigue and impact can easily result in gear fault. The running state of gear drive, which is a key component of the vehicle gearbox, can affect the service performance and driving safety of the entire vehicle. Real-time monitoring of gear state guarantees high-efficiency safety operation of vehicles. Gear engagement at fault may change the amplitude and phase of vibration signals. As vibration signals cover characteristic information of gear faults, collecting gearbox vibration signals is a major method in monitoring gear state [1]. Vibration signals collected on site often contain periodic harmonic signal and abundant noise interferences. These signals are generally nonlinear and non-stationary. Therefore, extracting gear fault characteristic components through the original vibration signal becomes the key of equipment monitoring.

Short-time Fourier transform and wavelet transform are traditional time-frequency analysis methods of vibration signals. As these approaches use fixed basis functions and lack adaptation to multi-component vibration signals, the

\footnotetext{
*E-mail address: wyije823@163.com

ISSN: 1791-2377 @ 2018 Eastern Macedonia and Thrace Institute of Technology. All rights reserved.

doi:10.25103/jestr.114.09
}

results of signal decomposition without physical meaning cannot be used to sift fault characteristic components [2]. Based on analysis of multi-component vibration signals, adaptive time-frequency analysis methods, such as signal sparse representation, empirical mode decomposition (EMD), and variational mode decomposition, were developed. Techniques based on signal sparse representation must solve the problem of establishing overcomplete dictionaries. The establishment of overcomplete dictionaries can directly influence the decomposition effect of signals [3]. Decomposition based on EMD does not need to establish the overcomplete dictionaries and basis functions because it decomposes multi-component signals into single-component signals by adaptively selecting bandwidth according to characteristics of the analyzed signal. However, decomposition based on EMD has many disadvantages, such as lack of strict mathematical basis, modal aliasing, and end effect [4][5][6]. Dragomiretskiy [7] proposed non-recursion adaptive variational mode decomposition (VMD), which transformed the signal decomposition problem into a constraint optimization problem and decomposed the multicomponent signal into intrinsic mode functions (IMFs) of different frequencies. Although this method possesses a strict mathematical basis, VMD only performs complete decomposition of the signal; thus, determining the number of decomposed modes $\mathrm{k}$ and sifting the fault characteristic component are difficult. Therefore, rapidly selecting fault characteristic components from IMFs and determining the 
gear fault state during VMD of multi-component vibration signals are problems that must be urgently solved.

In this study, VMD of multi-component vibration signals of gear fault is conducted, and corresponding influencing factors are analyzed. The selection method of fault characteristic component is optimized to determine fault characteristic components adaptively at signal decomposition. On this basis, characteristic frequency of fault can be extracted. Research results provide references for real-time determination of gear state.

\section{State of the art}

Many studies on gear fault diagnosis have been published worldwide. Accurate extraction of fault characteristic components is one of the main problems in fault diagnosis. He et al. [8] eliminated resonant harmonic components and noises in the original vibration signals by resonance-based sparse signal decomposition. Additionally, they strengthened period pulse signals in low-resonance components through the maximum correlation kurtosis deconvolution method and extracted fault characteristic components of multicomponent vibration signals. However, this approach acquired fault characteristic components in two steps, thereby increasing the computation complexity. Wu et al. [9] implemented synchronous decomposition of multi-channel vibration signals by using multi-element EMD and reconstructed IMFs containing fault information according to the kurtosis criterion and correlation coefficient, respectively. Based on IMFs, their approach successfully obtained fault characteristic components of gear and rolling bearing and solved EMD mode mixing and the calibration problem of multi-channel signal modes. Although their method showed strong adaption, it required many acceleration sensors, thereby increasing the hardware cost and arrangement difficulty. VMD has been widely used in signal decomposition and reconstruction and fault diagnosis since it was proposed in 2014. VMD can decompose multicomponent signals into several IMFs from low to high frequency. The contrastive study on the effects of VMD and empirical mode decomposition method in multi-component vibration signals revealed that the IMFs obtained from VMD had a low degree of mode mixing and strong adaption to decomposition of strong noise signals [10][11]. However, VMD had to appoint the number of decomposed modes $\mathrm{k}$ in advance and failed to directly extract fault characteristic components. Jiang et al. [12] screened and reconstructed IMFs from VMD by using the correlation coefficient and utilized the reconstructed signal as data source for bearing fault diagnosis. This reconstructed signal was compared with that provided by denoising based on empirical mode decomposition, which proved the superiority of VMD. Nevertheless, correlation coefficients can only express linear relationships of variables but cannot reflect nonlinear relationships. Qian et al. [13] screened IMFs related to the original vibration signal for signal reconstruction via the mutual information method that can quantitatively express mutual dependence of two random variables. However, IMFs were screened to eliminate signal noise in the decomposition and reconstruction of the original vibration signal through VMD, which also had to cooperate with other methods to extract the fault characteristic components and determine the fault state. Influences of $\mathrm{k}$ on denoising effect were overlooked. Liu et al. [14] suggested the determination of $\mathrm{k}$ by detrended fluctuation analysis (DFA) during signal denoising via VMD. However, DFA was mainly applied to eliminate Gaussian white noise signals. Liu et al. [15] improved the condition for iteration termination of VMD based on mutual information method and adaptively determined $\mathrm{k}$. However, this approach did not extract fault characteristic components at signal decomposition but implemented demodulation analysis on each IMF by using the Teager energy operator and determined fault state of rotor by combining rub-impact fault characteristics. Tang et al. [16] proposed the optimization of VMD influencing factors by using particle swarm optimization (PSO) algorithm. They decomposed the fault vibration signal into several IMFs, and the component with minimum envelope entropy was used as the optimal characteristic component. Bearing fault characteristic components were directly extracted during signal decomposition. However, influences prior to setting parameter values in the PSO algorithm on VMD results were neglected.

The preceding studies mainly reconstruct signals by signal decomposition and then extract fault characteristic components and determine fault state by different methods. This approach increases the difficulty of extracting fault characteristic components. A few studies that determine fault characteristic component during signal decomposition are available. In the present study, the number of decomposed modes $\mathrm{k}$ and influences of signal noise on decomposition of multi-component vibration signals were discussed. Then, the selection method of gear fault characteristic component and the number of decomposed modes $\mathrm{k}$ were analyzed according to the relationship between gear mesh frequency and center frequency of decomposed IMFs. Hilbert envelope analysis on characteristic component of gear fault was conducted, and characteristic frequency of gear fault was proposed. This approach provided a new idea for gear fault diagnosis. In determining the fault characteristic component, each IMF and the original vibration signal were used to calculate the correlation coefficient, which was compared with the proposed technique in this study.

The rest of this study is organized as follows. Section 3 introduces algorithm steps of gear fault characteristic component selection and fault diagnosis based on the VMD algorithm and Hilbert envelope demodulation principle, respectively. Section 4 analyzes the simulation signal and experimental vibration signal of gear fault by using VMD. The characteristic component of gear fault is also determined, and the fault characteristic frequency is extracted to determine the gear fault state. Section 5 provides conclusions.

\section{VMD and Hilbert envelope demodulation}

\subsection{VMD}

The basic principle of VMD is to decompose multicomponent signal into the sum of limited IMFs. Based on this constraint, different IMFs are hypothesized to have limited bandwidth of different central frequencies. These IMFs are characteristic of sparsity, and the sum of estimated bandwidth of IMFs is the minimum. Signal decomposition based on EMD and VMD facilitates decomposition of complicated signals into different IMFs. Essentially, IMFs are amplitude-frequency demodulation signals expressed as follows: 
$u_{k}(t)=A_{k}(t) \cos \left(\phi_{k}(t)\right)$

where $u_{k}(t)$ represents IMFs after decomposition. The envelope is $A_{k}(t) \geq 0$, and the instant frequency is $\phi_{k}^{\prime}(t) \geq 0$. In addition, the variation of $A_{k}(t)$ and $\phi_{k}^{\prime}(t)$ is smoother than the phase position $\phi_{k}(t)$. In other words, IMFs can be used to express the pure harmonic signals comprising $A_{k}(t)$ and $\phi_{k}^{\prime}(t)$.

The VMD problem mainly involves establishing and solving the variation model. In establishing the variation model, the analytic signal of each IMF component $u_{k}(t)$ is calculated through Hilbert transform, and the unilateral spectrum is calculated. Then, the adjustment term of $e^{-j \omega_{k} t}$ is multiplied by Hilbert transform results, and the frequency spectrum of each IMF is adjusted to its corresponding baseband spectrum. Third, bandwidths of different IMFs are estimated by the squared L2 norm of the gradient. The constructed constraint variation model is expressed in Eq. (2). In other words, each IMF component is solved under the constraint that the sum of each IMF component $u_{k}(t)$ is equal to the original signal $f(t)$. Simultaneously, the sum of all IMF estimated bandwidth is the minimum.

$$
\begin{aligned}
& \min _{\left\{u_{k}\right\},\left\{\omega_{k}\right\}}\left\{\sum_{k=1}^{K}\left\|\partial_{t}\left[\left(\delta(t)+\frac{j}{\pi t}\right) * u_{k}(t)\right] e^{-j \omega_{k} t}\right\|_{2}^{2}\right\} \\
& \text { s.t. } \sum_{k=1}^{K} u_{k}(t)=f(t)
\end{aligned}
$$

where $u_{k}, \omega_{k}$ are the decomposed $\mathrm{k}$ IMFs and corresponding central frequencies $(k=1,2 \cdots, \mathrm{K}$ is the number of decomposed modes). $\delta(t)$ is the Drac distribution, and * indicates convolution.

Adaptive signal decomposition can be achieved by solving this constraint variation model. Here, the quadratic penalty term $\alpha$ and Lagrangian multiplier $\lambda$ are used to assure the reconstruction accuracy and the strict enforcement of the constraint and introduce the augmented Lagrangian $L$, respectively. The minimization problem in Eq. (2) is transformed into the unconstrained model, which can be expressed as follows:

$$
\begin{aligned}
& L\left(\left\{u_{k}\right\},\left\{\omega_{k}\right\}, \lambda\right):= \\
& \alpha \sum_{k=1}^{K}\left\|\partial_{t}\left[\left(\delta(t)+\frac{j}{\pi t}\right) * u_{k}(t)\right] e^{-j \omega_{k} t}\right\|_{2}^{2}+ \\
& \left\|f(t)-\sum_{k=1}^{K} u_{k}(t)\right\|_{2}^{2}+\left\langle\lambda(t), f(t)-\sum_{k=1}^{K} u_{k}(t)\right\rangle
\end{aligned}
$$

Saddle point of the augmented Lagrangian can be determined by alternating direction method of multipliers, which is the solution used in Eq. (2). This method extracts IMFs and corresponding central frequencies by continuously updating $u_{k}(t)$ and $\omega_{k}$ and demodulates IMFs to the corresponding baseband spectra. The relevant solving process mainly covers minimization problems of $u_{k}(t)$ and $\omega_{k}[7]$.

When updating $u_{k}(t)$, Eq. (3) can be rewritten into the following minimization problem:

$$
\begin{aligned}
& u_{k}^{n+1}=\arg \min _{u_{k} \in X}\left\|\partial_{t}\left[\left(\delta(t)+\frac{j}{\pi t}\right) * u_{k}(t)\right] e^{-j \omega_{k} t}\right\|_{2}^{2} \\
& +\left\|f(t)-\sum_{i} u_{i}(t)+\frac{\lambda(t)}{2}\right\|_{2}^{2}
\end{aligned}
$$

Based on the Parseval/Plancherel Fourier isometry under the $L^{2}$ norm, Eq. (4) can be solved by Eq. (5) in the frequency domain as follows:

$$
\begin{aligned}
& \hat{u}_{k}^{n+1}=\underset{u_{k}, u_{k} \in X}{\arg \min }\left\{\alpha \|\left. j \omega\left[\left(1+\operatorname{sgn}\left(\omega+\omega_{k}\right)\right) \hat{u}_{k}\left(\omega+\omega_{k}\right)\right]\right|_{2} ^{2}\right\} \\
& +\left\|\hat{f}(\omega)-\sum_{i} \hat{u}_{i}(\omega)+\frac{\hat{\lambda}(\omega)}{2}\right\|_{2}^{2}
\end{aligned}
$$

The solution in the frequency domain can be obtained by solving Eq. (5), which is expressed by Eq. (6). Inverse Fourier transform of Eq. (6) is performed, and the real part is the IMF of time domain.

$\hat{u}_{k}^{n+1}(\omega)=\frac{\hat{f}(\omega)-\sum_{i \neq k} \hat{u}_{i}(\omega)+\frac{\hat{\lambda}(\omega)}{2}}{1+2 \alpha\left(\omega-\omega_{k}\right)^{2}}$

Only the bandwidth prior is present; thus, no center frequency $\omega_{k}$ is found in the reconstructed fidelity term, and the minimization of $\omega_{k}$ can be rewritten as follows:

$$
\omega_{k}^{n+1}=\arg \min _{\omega_{k}}\left\{\left\|\partial_{t}\left[\left(\delta \mathrm{t}+\frac{j}{\pi t}\right) * u_{k}(t)\right] e^{-j \omega_{k} t}\right\|_{2}^{2}\right\}
$$

Similarly, the problem of center frequency in the frequency domain can be solved as follows:

$$
\omega_{k}^{n+1}=\frac{\int_{0}^{\infty} \omega\left|\hat{u}_{k}(\omega)\right|^{2} d \omega}{\int_{0}^{\infty}\left|\hat{u}_{k}(\omega)\right|^{2} d \omega}
$$

According to the minimization problem solution of Eqs. (5) and (7) and the alternating direction method of multipliers, the complete VMD algorithm can be obtained. Specific steps are shown as follows:

1) Initialize $\left\{\hat{u}_{k}^{1}\right\},\left\{\omega_{k}^{1}\right\}, \hat{\lambda}^{1}, n \leftarrow 0$.

2) Execute the main cycle: $n \leftarrow n+1$.

3) Execute the first cycle: for $k=1: K$, update $\hat{u}_{k}$ when $\omega \geq 0$. 


$$
\hat{u}_{k}^{n+1}(\omega) \leftarrow \frac{\hat{f}(\omega)-\sum_{i<k} \hat{n}_{i}^{n+1}(\omega)-\sum_{i>k} \hat{u}_{i}^{n}(\omega)+\frac{\hat{\lambda}^{n}(\omega)}{2}}{1+2 \alpha\left(\omega-\omega_{k}^{n}\right)^{2}}
$$

Update $\omega_{k}$ as follows:

$$
\omega_{k}^{n+1} \leftarrow \frac{\int_{0}^{\infty} \omega\left|\hat{u}_{k}(\omega)\right|^{2} d \omega}{\int_{0}^{\infty}\left|\hat{u}_{k}(\omega)\right|^{2} d \omega}
$$

End the cycle when $k=K$.

4) When $\omega \geq 0$, update $\hat{\lambda}$ by dual ascent:

$$
\hat{\lambda}^{n+1}(\omega) \leftarrow \hat{\lambda}^{n}(\omega)+\tau\left(\hat{f}(\omega)-\sum_{k} \hat{u}_{k}^{n+1}(\omega)\right)
$$

5) Repeat steps (2) to (4) until the convergence condition $\sum_{k=1}^{K}\left\|\hat{u}_{k}^{n+1}-\hat{u}_{k}^{n}\right\|_{2}^{2} /\left\|\hat{u}_{k}^{n}\right\|_{2}^{2}<\varepsilon$ is met.

\subsection{Hilbert envelope demodulation}

Hilbert envelope demodulation is an effective approach to extract modulation information of fault signal. Based on Hilbert transform, the envelope demodulation of narrowband signal was conducted to solve the instantaneous frequency. Hilbert transform of real signal $x(t)$ is expressed as:

$$
H[x(t)]=\tilde{x}(\mathrm{t})=\frac{1}{\pi} \int_{-\infty}^{\infty} \frac{s(\tau)}{t-\tau} d \tau
$$

where signals $\tilde{x}(t)$ and $x(t)$ are phase spectrum difference, whereas the amplitude spectra are exactly the same. The two signals are orthogonal. Therefore, the new analytical signal can be rewritten as follows:

$$
z(t)=x(t)+j \tilde{x}(t)
$$

The envelope $A(t)$ of analytical signal is defined as

$$
A(t)=\sqrt{x(t)^{2}+\tilde{x}(t)^{2}}
$$

Hilbert envelope spectra are the spectrum analysis of the analytical signal envelope $A(t)$.

\subsection{Steps of gear fault diagnosis}

When the gear on the fixed axis is operating normally, the vibration signals produced by gear engagement mainly include gear mesh frequency and its harmonic components. A fixed multiple relationship exists between gear mesh frequency and spindle speed. When gear fault occurs, the fault gear engages with the periodic rotation of the gear shaft. At this moment, the vibration signals of gear not only cover gear mesh frequency but also generate side band at the gear mesh frequency due to the modulation on rotating frequency of fault gear axis and gear mesh frequency and their frequency multiplication. Thus, multi-component amplitude and frequency modulation signals are generated [17]. This finding reflects that the key of gear fault feature extraction is to extract side band of gear mesh frequency from gear fault vibration signals.

According to the analysis of simulated and experimental signals, the quadratic penalty term $\alpha$ of VMD in this study used the sampling frequency [18]. Based on VMD of the original vibration signal, the relationship between center frequencies of decomposed IMFs and gear mesh frequency is analyzed. The fault characteristic component and $\mathrm{k}$ are determined by the adaptive method. Hilbert envelope demodulation analysis of the fault characteristic component is conducted through which fault characteristic frequency is obtained. On this basis, the gear fault state is determined. The gearbox fault diagnosis steps based on VMD and envelope spectra are as follows:

(1) Gear mesh frequency $\omega_{m}$ is calculated by prior information.

(2) Given the determined quadratic penalty term $\alpha, \mathrm{k}$ is successively increased from 2 during VMD of the original vibration signal. The center frequencies $\omega_{k}$ of the decomposed IMFs and gear mesh frequency $\omega_{m}$ are compared. When the frequency difference is less than a certain threshold, this IMF is chosen as the fault characteristic component and the iterative decomposition is terminated. At this moment, $\mathrm{k}$ is adaptively determined.

(3) Envelope spectrum of fault characteristic component is obtained from Hilbert demodulation. If the rotating frequency of the gear shaft is observed, then this gear is in a faulty condition.

\section{Result analysis and discussion}

\subsection{Simulation analysis}

In actual gear transmission, the amplitude modulation (AM) and frequency modulation (FM) produced by the rotating frequency of the shaft upon occurrence of gear fault exist simultaneously. The simulation signal $y(t)$ of the gear fault is expressed in Eq. (15) to verify the performance of the proposed method in gear fault diagnosis. This signal has AM signal $y_{0}(t)$, FM signal $y_{1}(t)$, and AM/FM signal $y_{2}(t)$. The signal $n(t)$ is Gaussian white noise. The rotating axis frequency lies in the simulated fault gear in $f(t)=15 \mathrm{~Hz}$. The total simulation signal at the gear fault is:

$$
\begin{aligned}
y_{0}(t)= & (1.2+\cos (2 \pi 15 t)) * \cos (2 \pi 50 t) \\
y_{1}(t)= & \cos (2 \pi 240 t+\cos (2 \pi 15 t)) \\
y_{2}(t)= & (1+0.8 \cos (2 \pi 15 t)) * \\
& \cos (2 \pi 480 t+0.7 \cos (2 \pi 15 t)) \\
y(t)= & y_{0}(t)+y_{1}(t)+y_{2}(t)+n(t)
\end{aligned}
$$

The time-domain waveform of simulated signal $y(t)$ without noise $n(t)$ is shown in Fig. 1(a). VMD must determine the value of $\mathrm{k}$ in advance. In this study, a multi- 
component signal comprising three component signals is known; therefore, VMD starts from $\mathrm{k}=3$. The time-domain waveforms of IMFs of the simulation signal $y(t)$ when $\mathrm{k}=$ 3-4 under $n(t)=0$ are shown in Fig. 2. The time-domain waveforms of IMFs when $\mathrm{k}=3$ are shown in Fig. 2(a), where AM signal $y_{0}(t)$, FM signal $y_{1}(t)$, and AM/FM signal $y_{2}(t)$ are effectively decomposed. The time-domain waveforms of IMFs when $\mathrm{k}=4$ are shown in Fig. 2(b). IMF3 and IMF4 are similar when $\mathrm{k}$ value is set high, indicating excessive decomposition. Center frequencies of IMFs decomposed by VMD when $\mathrm{k}=3-6$ are shown in Table 1. Signals can be sparsely decomposed when $\mathrm{k}=3$. The center frequency $\omega_{k}$ of decomposed IMFs agrees well with the carrier frequencies $(50,240$, and $460 \mathrm{~Hz})$ of a given signal. High-frequency component introduces certain errors. When $\mathrm{k}$ is higher than the number of a given signal (3), high-frequency components in the center frequency of decomposed IMFs are relatively close to one another, indicating excessive decomposition. Therefore, $\mathrm{k}$ can considerably influence the performance of VMD.

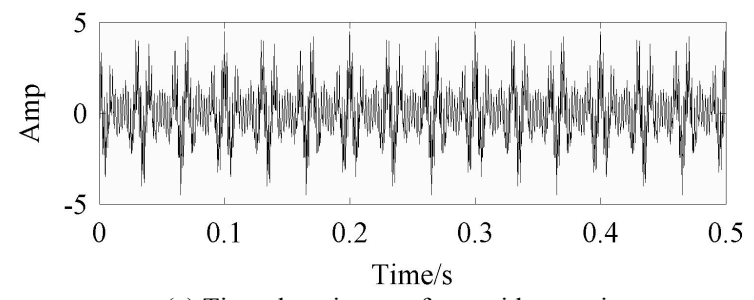

(a) Time-domain waveform without noise

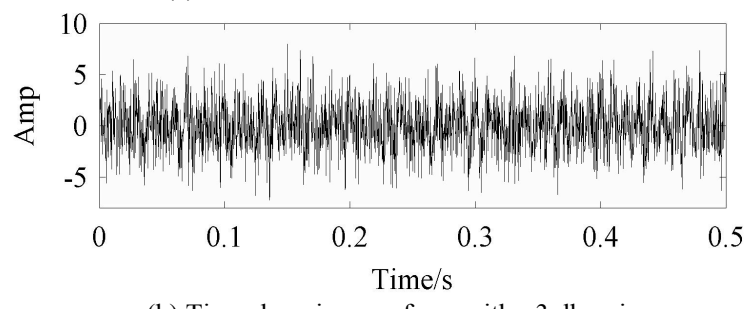

(b) Time-domain waveform with $-3 \mathrm{db}$ noise

Fig. 1. Time-domain waveform of simulated signal $y(t)$
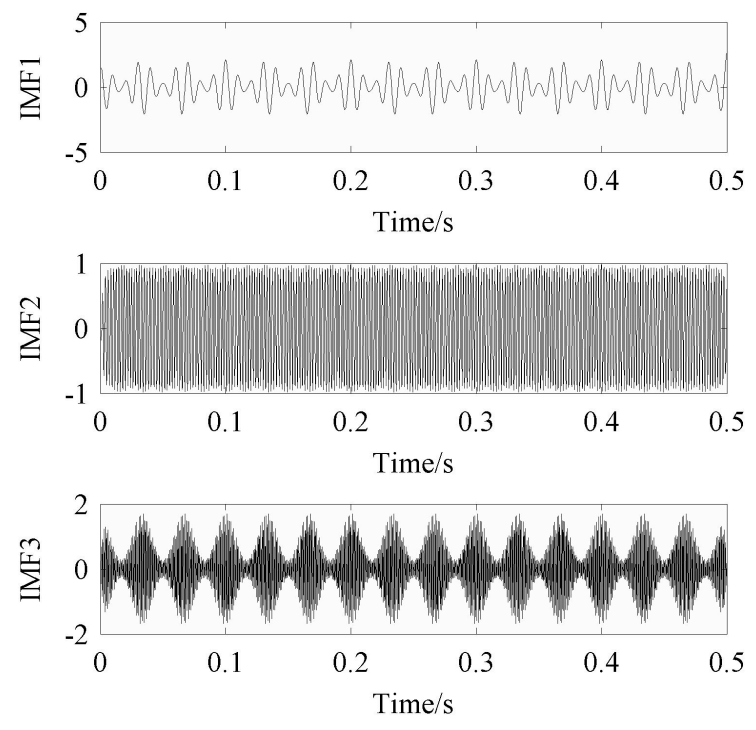

(a) Time-domain waveform of decomposed IMFs when $\mathrm{k}=3$
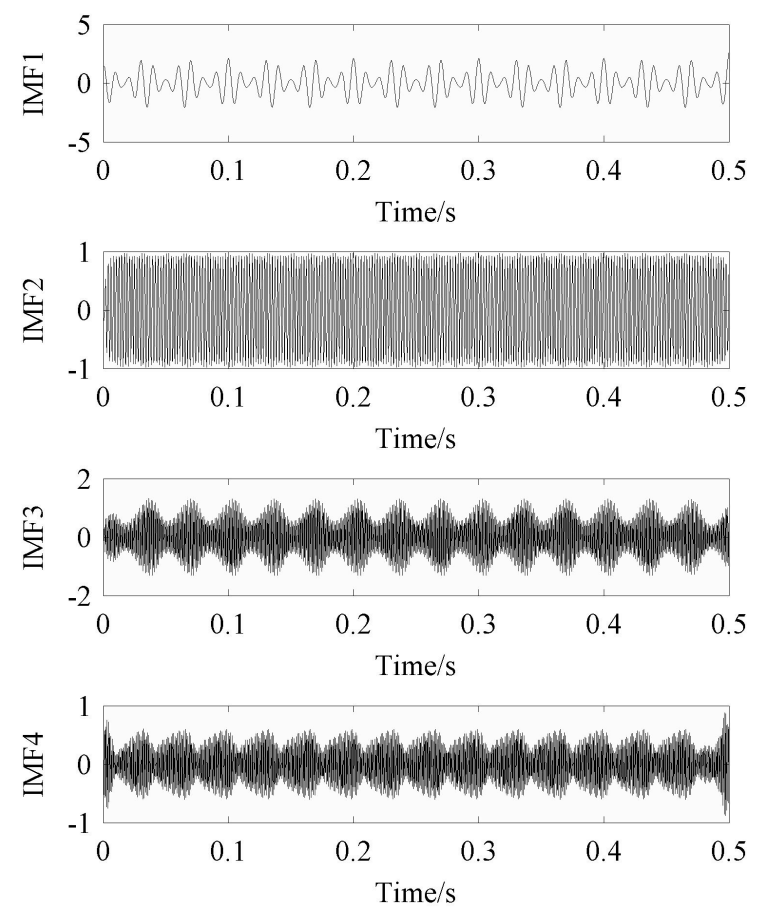

(b) Time-domain waveform of decomposed IMFs when $\mathrm{k}=4$ Fig. 2. Time-domain waveform of different IMFs without noise

Table 1. Center frequencies of IMFs without noise when $\mathrm{k}=$

\begin{tabular}{l|l|l|l|l|l|l}
$3-6$ & \multicolumn{5}{c}{ Center frequencies $\omega_{k}(\mathbf{H z})$} \\
\hline $\mathbf{k}$ & \multicolumn{6}{c}{. } \\
\hline 3 & 49.9 & 239.8 & 480.0 & & & \\
4 & 49.9 & 239.8 & 477.3 & 495.7 & & \\
5 & 49.9 & 239.8 & 477.6 & 494.2 & 508.6 & \\
6 & 49.9 & 239.7 & 448.1 & 480.0 & 496.4 & 464.6 \\
\hline
\end{tabular}

The time-domain waveforms of the simulated signal $y(t)$ with $-3 \mathrm{db}$ Gaussian white noise signal $n(t)$ are shown in Fig. 1(b). The time-domain waveforms of decomposed IMFs when $\mathrm{k}=3-4$ are shown in Fig. 3. Center frequencies $\omega_{k}$ of decomposed IMFs when $\mathrm{k}=3-5$ are listed in Table 2. These frequencies are compared with the given carrier frequency. The simulated signal cannot be accurately decomposed when $\mathrm{k}=3$. When $\mathrm{k}=4-5$, the center frequency is higher than the given carrier frequency. According to the contrastive analysis decomposition results without noise, the high center frequency is due to influences of noise signal on the VMD performance. In this condition, the original multi-component signal can still be accurately decomposed.

Table 2. Center frequencies of IMFs with $-3 \mathrm{db}$ noise and $\mathrm{k}$

\begin{tabular}{l|l|l|l|l|l}
$=3-4$ & \multicolumn{5}{c}{ Center frequencies $\omega_{k}(\mathbf{H z})$} \\
\hline $\mathbf{k}$ & \multicolumn{5}{c}{} \\
& 52.7 & 478.9 & 673.3 & & \\
4 & 49.5 & 244.7 & 481.9 & 843.5 & \\
5 & 52.7 & 239.2 & 480.4 & 706.8 & 957.0 \\
\hline
\end{tabular}

In actual applications, the composition of multicomponent signal is often unknown, causing difficulty in determining $\mathrm{k}$. No effective technique is available to determine $\mathrm{k}$ yet. The ultimate objective of this study is to extract the characteristic components of gear fault and 
determine the gear fault state. According to the analysis of VMD of simulated signal, the selection method of characteristic components of the gear fault is introduced with consideration of prior structural information of the gear set. Additionally, the condition to terminate iteration is improved and $\mathrm{k}$ is simultaneously determined. Given the number of teeth and rotating speed of the gear shaft, the gear mesh vibration frequency can be calculated. When the center frequency of one IMF from VMD of the vibration signal is close to the gear mesh frequency, $\mathrm{k}$ is not increased for decomposition. IMF of this center frequency is used as the characteristic component of the gear fault for Hilbert envelope demodulation and determination of gear fault state. Table 2 shows that if the gear mesh frequency in the original synthesis signal is $50 \mathrm{~Hz}$, then only the $50 \mathrm{~Hz}$ signal component is decomposed when $\mathrm{k}=3$, whereas 240 and 460 $\mathrm{Hz}$ components are not decomposed. The Hilbert envelope demodulation of the IMF with $50 \mathrm{~Hz}$ of center frequency is shown in Fig. 4. The rotating frequency of the shaft where the fault gear is located can be tested by $15 \mathrm{~Hz}$. Therefore, faults can be detected from this gear.
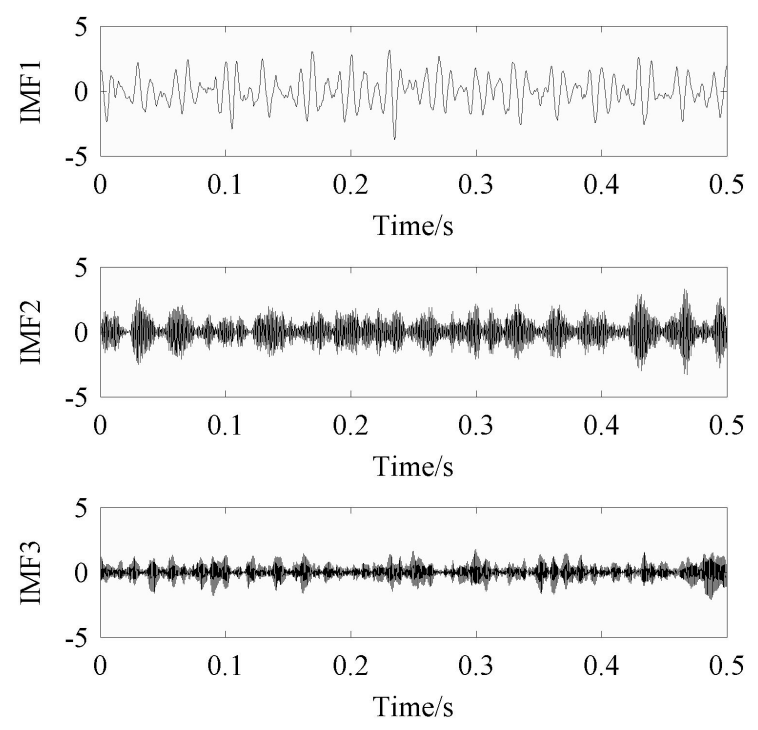

(a) Time-domain waveforms of decomposed IMFs when $\mathrm{k}=3$
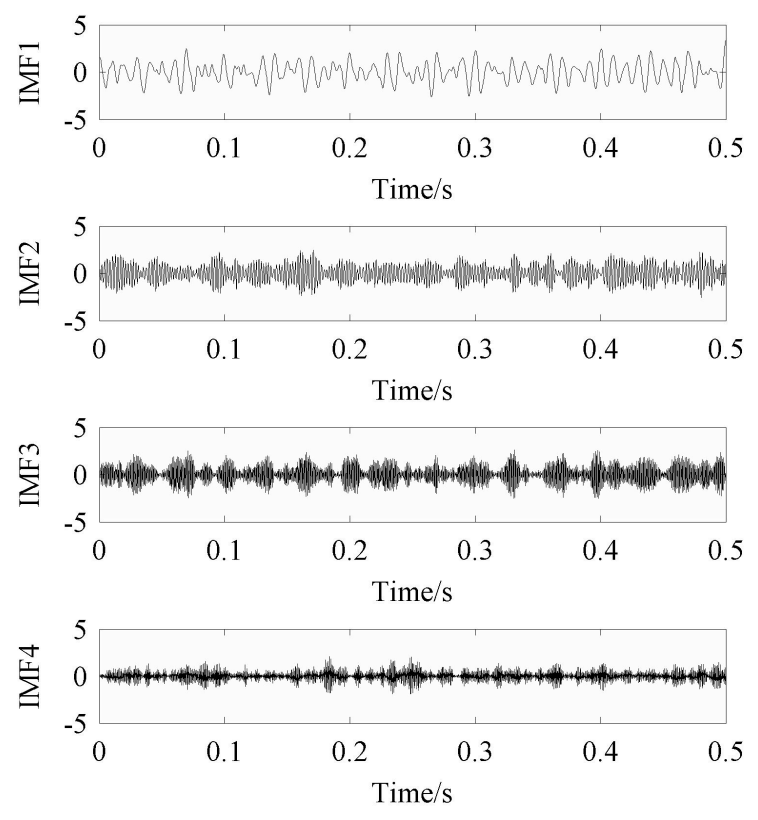

(b) Time-domain waveforms of decomposed IMFs when $\mathrm{k}=4$

Fig. 3. Time-domain waveforms of decomposed IMFs with $-3 \mathrm{db}$ noise

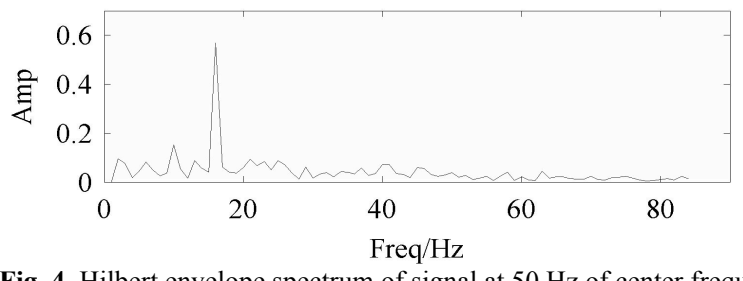

Fig. 4. Hilbert envelope spectrum of signal at $50 \mathrm{~Hz}$ of center frequency

\subsection{Analysis of experimental signal}

Gear fault data are analyzed on the test table of rotating mechanical vibration to verify the validity of the proposed method based on VMD and envelope spectral analysis in actual gear fault diagnosis. The test table is equipped with speed variator transducer, motor of driving system, gearbox, revolution speed transducer, acceleration sensor, and magnetic powder brake. The gearbox has one group of teeth that engage into the single-level driving. The number of big and small teeth is 75 and 55, respectively, and gear modulus is 2 . The revolution speed transducer measures the rotating speed of the small teeth, while the acceleration sensor measures the vibration signal of gearbox bearing block. The sampling frequency is $5120 \mathrm{~Hz}$, and the sampling time is $1 \mathrm{~s}$. When the actual rotating speed of the small gear is $840 \mathrm{r} / \mathrm{min}$, vibration signal collected under one broken tooth of big gear is shown in Fig. 5(a).

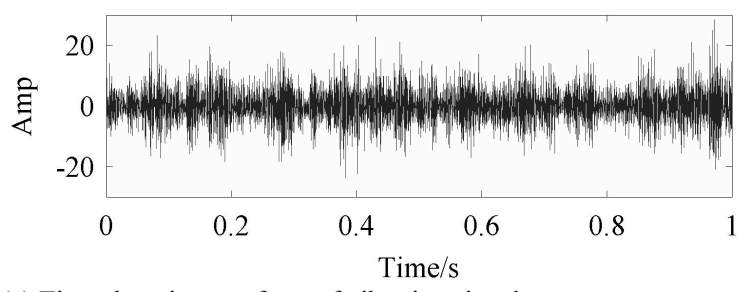

(a) Time-domain waveform of vibration signal

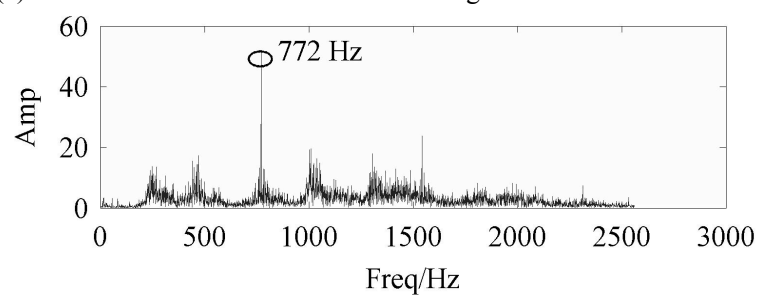

(b) Frequency spectrum of vibration signal

Fig. 5. Vibration signal of one broken tooth of big gear

In this experiment, the mesh frequency of this gear set is calculated as $770 \mathrm{~Hz}$. The rotating frequency of the shaft where the fault gear is located is $10 \mathrm{~Hz}$. The frequency spectrum of the gear vibration signal is shown in Fig. 5(b). The frequency at maximum amplitude is $772 \mathrm{~Hz}$, which is approximately equal to the gear mesh frequency $(770 \mathrm{~Hz})$. This finding is due to the maximum energy at gear engagement.

VMD of the vibration signal at snaggletooth fault of gear is conducted. The center frequencies of IMFs in each group when $\mathrm{k}=2-4$ are listed in Table 3 .

Table 3. Center frequencies of IMFs when $\mathrm{k}=2-4$

\begin{tabular}{l|l|l|l|l}
\hline $\mathbf{k}$ & \multicolumn{4}{|c}{ Center frequencies $\omega_{k}(\mathbf{H z})$} \\
\hline 2 & 276 & 1337 & & \\
3 & 274 & 776.4 & 1339 & \\
4 & 273.9 & 776.4 & 1337 & 1905 \\
\hline
\end{tabular}


IMFs from VMD and the corresponding Hilbert envelope spectra when $\mathrm{k}=2$ are shown in Fig. 6. The frequencies of IMF1 and IMF2 are 276 and $1337 \mathrm{~Hz}$, respectively. The envelope spectra of two IMFs in Fig. 6(b) are compared. The fault characteristic frequency of $10 \mathrm{~Hz}$ is not shown in the first image. Table 3 shows that the center frequency of IMF is $276 \mathrm{~Hz}$, which is unrelated to the gear mesh and rotating frequency of the gear shaft. In the second image, the rotating frequency $(10 \mathrm{~Hz})$ of the shaft where the fault gear is located can be identified, but the fault characteristic frequency is insignificant. At this moment, the center frequency of IMF is $1337 \mathrm{~Hz}$, which is approximately twice the gear mesh frequency.
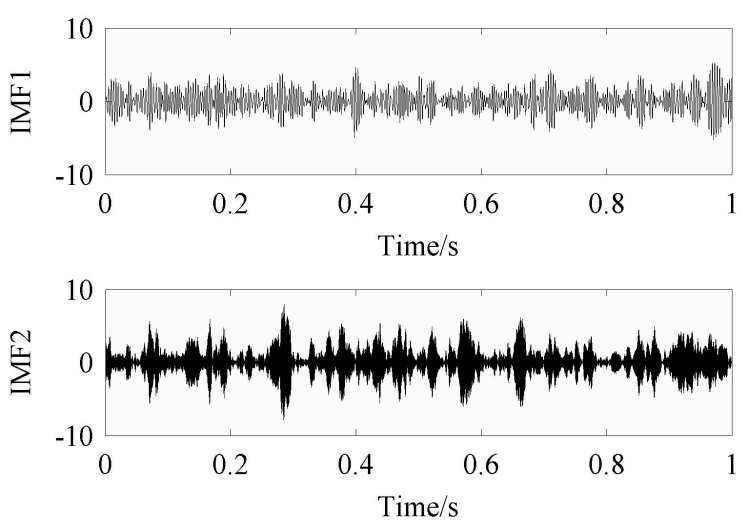

(a) IMFs
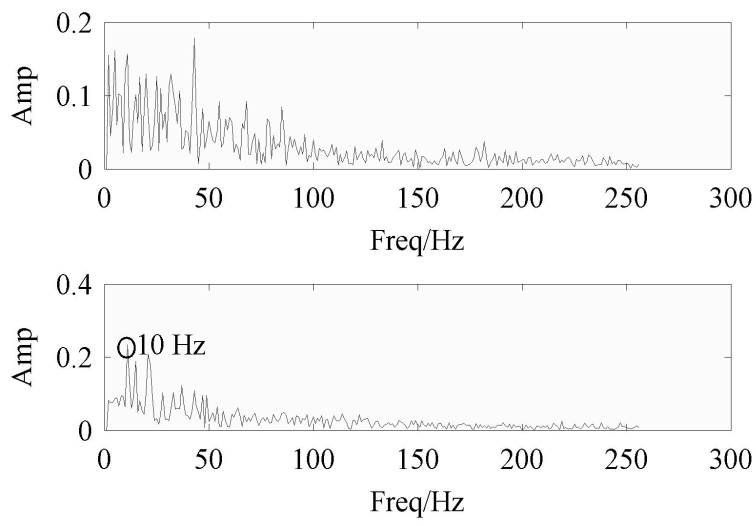

(b) Hilbert envelope spectra

Fig. 6. IMFs and corresponding Hilbert envelope spectra when $\mathrm{k}=2$
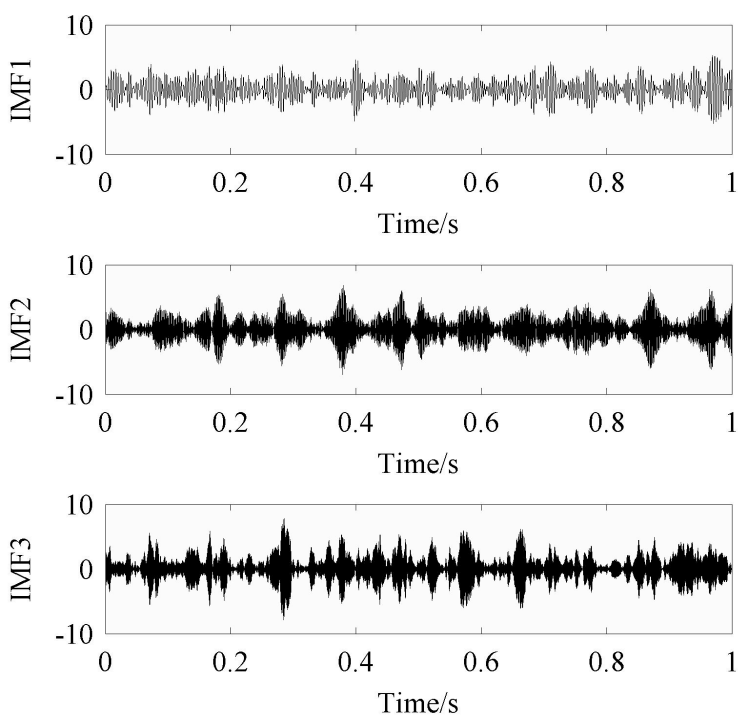

(a) IMFs
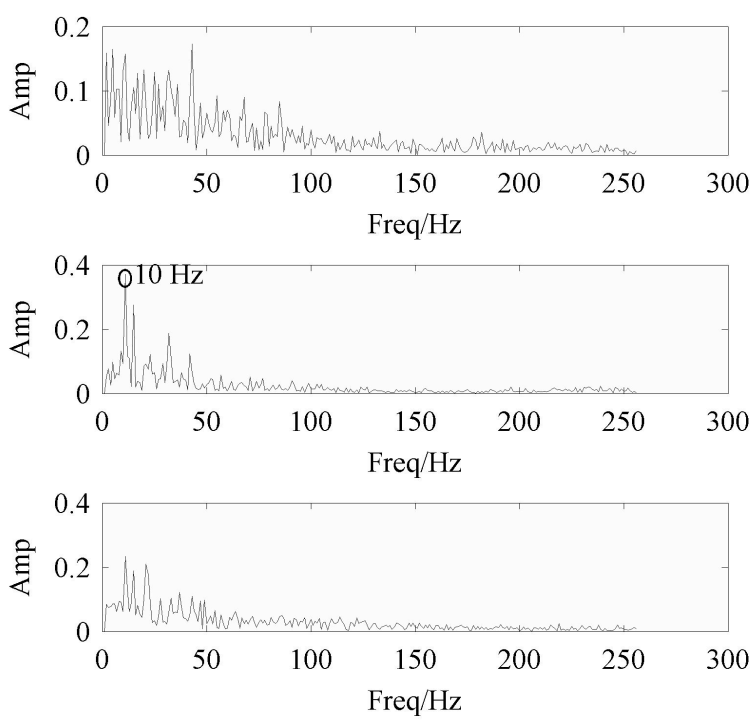

(b) Hilbert envelope spectra

Fig. 7. IMFs and Hilbert envelope spectra when $\mathrm{k}=3$

IMFs from VMD and the corresponding Hilbert envelope spectra when $\mathrm{k}=3$ are shown in Fig. 7. The frequencies of IMF1, IMF2, and IMF3 are 274, 776.4, and $1339 \mathrm{~Hz}$, respectively. In Fig. 7(b) and Table 3, the envelope spectrum when the center frequency is $776.4 \mathrm{~Hz}$ show that the rotating frequency of shaft where the fault gear is located is $10 \mathrm{~Hz}$, indicating the existence of gear fault. The envelope spectrum when the center frequency is $1339 \mathrm{~Hz}$ is similar to second image in Fig. 6(b), indicating that the fault frequency is insignificant and misjudgment is possible. Therefore, VMD is terminated when the difference between center frequency of one IMF and the fault gear mesh frequency is lower than a certain threshold. This IMF is determined as the characteristic component of gear fault. This approach can adaptively determine fault characteristic component and $\mathrm{k}$ during VMD of the vibration signal, thus enabling the implementation of Hilbert envelope demodulation of fault characteristic component. In addition, the fault characteristic frequency of this component is extracted and compared with the prior rotating frequency of the gear shaft to determine the fault state of the gear.

Table 4. Correlation coefficient between IMFs and original signal when $\mathrm{k}=2-4$

\begin{tabular}{l|l|l|l|l}
\hline $\mathbf{k}$ & \multicolumn{4}{|c}{ Correlation coefficient } \\
\hline 2 & 0.3807 & 0.5191 & & \\
3 & 0.3678 & 0.4307 & 0.5112 & \\
4 & 0.3673 & 0.4298 & 0.5074 & 0.1725 \\
\hline
\end{tabular}

In this study, the correlation coefficients between IMFs from VMD and the original signal are also calculated (Table 4). The third IMF shows the highest correlation coefficient when $\mathrm{k}=3-4$. In this case, the fault characteristic frequency of Hilbert envelope demodulation is insignificant. Therefore, the proposed means of selecting fault characteristic component is more effective than the correlation coefficient method.

\section{Conclusions}

This study analyzes VMD of the multi-component signal, selection of fault characteristic component, extraction of fault characteristic frequency, and determination of gear 
fault state by combining VMD and envelope spectral theory. The study also aims to explore adaption and validity of VMD to decomposition of the multi-component gear fault signal and extraction of fault characteristic component and achieve automatic selection of fault characteristic component and diagnosis of gear fault state. The simulated multi-component signal is compared with the gear fault signal on the test bed. The following major conclusions can be drawn:

(1) The number of decomposed modes (k) and signal noise influence extraction of IMFs are obtained from VMD and fault characteristic components, respectively.

(2) For multi-component vibration signal, the iteration termination conditions of VMD are improved by comparing the gear mesh frequency and center frequency of IMFs. Additionally, the characteristic component of gear fault is adaptively extracted.

(3) Hilbert envelope demodulation is conducted on fault characteristic components of simulated and experimental signals. The gear fault state is determined through comparison with the rotating frequency of shaft where the fault gear is located.

(4) The proposed approach is more effective than the correlation coefficient method in determining the fault characteristic component.

In this study, an adaptive selection technique of fault characteristic component is proposed by combining experimental and simulated signals. The termination conditions of VMD are improved, and the extraction of fault characteristic component is simplified. The research results provide new ideas for VMD applications in gear fault diagnosis. However, attention should be provided to gear fault diagnosis. Future studies can apply the proposed method in the diagnosis of gear and bearing composite fault.

This is an Open Access article distributed under the terms of the Creative Commons Attribution Licence

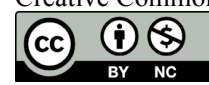

\section{References}

1. Meng, Z., Wang, Y. C., Hu, M., "Fault Feature Extraction Method of Gear Based on Improved Local Mean Decomposition and Instantaneous Energy Distribution-sample Entropy". Journal of Mechanical Engineering, 52(5), 2016, pp. 169-176.

2. Li, B. Q., "The Machinery Fault Diagnosis Method Based on Adaptive and Sparsest Time-Frequency Analysis". Doctoral Dissertation of Hunan University, China, 2016, pp.1-8.

3. He, G. L., Ding, K., Lin, H, B., "Matching Pursuit Method for Coupling Modulation Signal Separation of Gearbox Vibration". Journal of Mechanical Engineering, 52(1), 2016, pp.102-108.

4. Lei, Y., Kong, D., and Li, N.J.J.o.M.E., "Adaptive ensemble empirical mode decomposition and its application to fault detection of planetary gearboxes". Journal of Mechanical Engineering, 50(3), 2014, pp. 64-70.

5. Tang, H. B., Wu, H. G., Hua, G. J., Ma, C. X., "Fault diagnosis of pump using EMD and envelope spectrum analysis". Journal of Vibration and Shock, 31(9), 2012, pp.44-48.

6. Zhang, C., Chen, J. J., "A fault diagnosis method of roller bearing based on EMD De-noising and spectral kurtosis". Mechanical Science and Technology for Aerospace Engineering, 34(2), 2015, pp.44-48.

7. Dragomiretskiy, K., Zosso, D., "Variational Mode Decomposition". IEEE Transactions on Signal Processing, 62(3), 2014, pp.531-544

8. He, Q., Guo, Y. G., Wang, X., Ren, Z. H., Li, J. M., “Gearbox Fault Diagnosis based on RB-SSD and MCKD". China Mechanical Engineering, 28(13), 2017, pp. 1528-1534.

9. Wu, Z., Yang, S. P., Liu, Y. Q., "Rotating machinery early fault diagnosis method based on multivariate empirical mode decomposition". Chinese Journal of Scientific Instrument, 37(2), 2016, pp.241-248.

10. Li, Z. N., Zhu, M., "Research on Mechanical Fault Diagnosis Method based on Variational Mode Decomposition". Acta Armamentarii, 38(3), 2017, pp.593-599.
11. Tang, G., Luo, G. G., Zhang, W. H., Yang, C. J., "Underdetermined Blind Source Separation with Variational Mode Decomposition for Compound Roller Bearing Fault Signals". Sensors, 16 (6), 2016, pp.897.

12. Jiang, W. L., Wang, H. N., Zhu, Y., Wang, Z. W., "Integrated VMD Denoising and KFCM Clustering Fault Identification method of rolling bearing”. China Mechanical Engineering, 28(10), 2017, pp. 1215-1220.

13. Qian, L., Kang, M., Fu, X. Q., Wang, X. S., "Application of adaptive morphology in bearing fault diagnosis based on VMD". Journal of Vibration and Shock, 36(3), 2017, pp. 227-233.

14. Liu, Y. Y., Yang, G. L., Li, M., Yin, H. L., "Variational mode decomposition denoising combined the detrended fluctuation analysis". Signal Processing, 125(C), 2016, 349-364.

15. Liu, S. K., Tang, G. J., Wang, X. L., "Time-frequency Analysis Method of Rotating Mechanical Fault based on Improved Variational Mode Decomposition". Journal of Vibration Engineering, 29(6), 2016, 1119-1126.

16. Tang, G. J., Wang, X. L., "Parameter optimized variational mode decomposition method with application to incipient fault diagnosis of rolling bearing". Journal of Xi'an Jiaotong University, 49(5), 2015, 73-81.

17. Man, Z. H., Wang, W. Y., Khoo, S. Y., Yin, J. L., "Optimal sinusoidal modelling of gear mesh vibration signals for gear diagnosis and prognosis". Mechanical Systems and Signal Processing, 33(2), 2012, 256-274.

18. Lv, Z. L., "Research on Incipient Fault Diagnosis Methods for Rotating Machinery based on VMD and Optimized MSVM". Doctoral Dissertation of Chongqing University, China, 2016, pp.81-83. 